\title{
Oral Delivery of Probiotics Using pH-Sensitive Phthalyl Inulin Tablets
}

\author{
Whee-Soo Kim ${ }^{1 \dagger}$, Chong-Su Cho ${ }^{1 \dagger}$, Liang Hong ${ }^{1}$, Geon Goo Han ${ }^{1}$, Bum Ju Kil ${ }^{2}$, Sang-Kee Kang ${ }^{3}$, Dae-Duk Kim ${ }^{4}$, \\ Yun-Jaie Choi ${ }^{1 *}$, and Chul Sung Huh, ${ }^{3,5 *}$ \\ ${ }^{1}$ Department of Agricultural Biotechnology and Research Institute of Agriculture and Life Sciences, Seoul National University, Seoul 08826, \\ Republic of Korea \\ ${ }^{2}$ WCU Biomodulation Major and Center for Food and Bioconvergence, Seoul National University, Seoul 08826, Republic of Korea \\ ${ }^{3}$ Institute of Green-Bio Science E Technology, Seoul National University, Pyeongchang-gun 25354, Republic of Korea \\ ${ }^{4}$ College of Pharmacy and Research Institute of Pharmaceutical Sciences, Seoul National University, Seoul 08826, Republic of Korea \\ ${ }^{5}$ Graduate School of International Agricultural Technology, Seoul National University, Pyeongchang-gun 25354, Republic of Korea
}

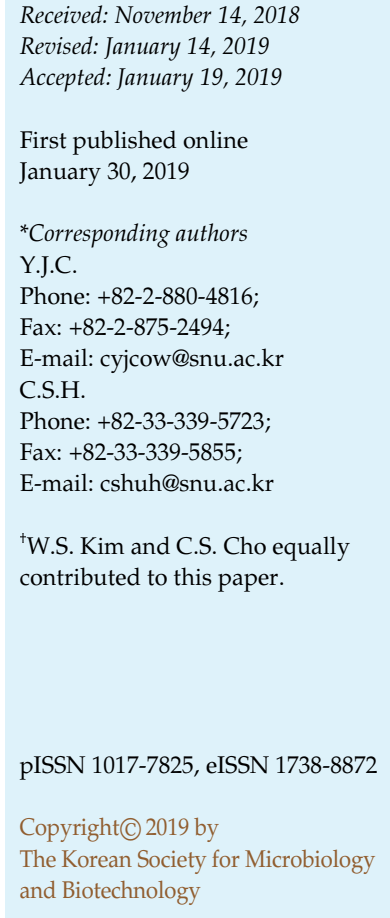

Probiotics show low cell viability after oral administration because they have difficulty surviving in the stomach due to low $\mathrm{pH}$ and enzymes. For the oral delivery of probiotics, developing a formula that protects the probiotic bacteria from gastric acidity while providing living cells is mandatory. In this study, we developed tablets using a new pH-sensitive phthalyl inulin (PI) to protect probiotics from gastric conditions and investigated the effects of different compression forces on cell survival. We made three different tablets under different compression forces and measured survivability, disintegration time, and kinetics in simulated gastric-intestinal fluid. During tableting, there were no significant differences in probiotic viability among the different compression forces although disintegration time was affected by the compression force. A higher compression force resulted in higher viability in simulated gastric fluid. The swelling degree of the PI tablets in simulated intestinal fluid was higher than that of the tablets in simulated gastric fluid due to the $\mathrm{pH}$ sensitivity of the PI. The probiotic viability formulated in the tablets was also higher in acidic gastric conditions than that for probiotics in solution. Rapid release of the probiotics from the tablet occurred in the simulated intestinal fluid due to the $\mathrm{pH}$ sensitivity. After 6 months of refrigeration, the viability of the PI probiotics was kept. Overall, this is the first study to show the $\mathrm{pH}$-sensitive properties of PI and one that may be useful for oral delivery of the probiotics.

Keywords: Probiotics, oral delivery, pH-sensitive tablet, phthalyl inulin

\section{Introduction}

Nowadays, probiotics are used as an alternative for antibiotics since they are generally considered as safe and confer health benefits to the host. Probiotics produce antimicrobial molecules (e.g., lactic acid and bacteriocins) and enzymes, enabling them to function as an alternative to antibiotics owing to their antimicrobial effects toward pathogens [1]. Among probiotics, Lactobacillus is the most common probiotic because Lactobacillus has shown excellent antimicrobial activity against Salmonella spp. and Escherichia coli, which are major pathogens in livestock animals $[2,3]$.
Lactobacillus reduces weight loss, improves feed intake and improves the growth performance of animals [4]. In our previous study, we isolated $L$. reuteri LRT18 from porcine feces and selected the highest antimicrobial effect on K88positive Escherichia coli and Salmonella enterica subsp. [5].

To deliver probiotics orally, many strategies target the intestine as a main location for the probiotics to provide the most beneficial effect to the host [6, 7]. Since the intestine has a neutral $\mathrm{pH}$, long transit time and reduced host enzymatic activity, an intestine-specific drug delivery system increases the bioavailability of probiotics [8]. However, oral delivery of probiotics is extremely challenging 
because they can get destroyed and/or cause cell death due to stomach acid [9]. Therefore, delivering probiotics to the intestine safely while passing through harsh gastric conditions is of utmost importance in enabling probiotics to provide their therapeutic effect to the host. Recently, polymeric delivery systems have been attracting attention as a means to deliver biological materials, proteins, genes, and chemotherapeutics because they can deliver drugs to target sites [10]. Among many strategies for orally delivering probiotics, $\mathrm{pH}$-sensitive polymers, such as hydroxypropyl methylcellulose phthalate (HPMCP) [11], hydroxypropylmethyl cellulose acetated succinate [12], and cellulose acetate phthalate (CAP) [13] have been used to protect probiotics from harsh gastric conditions since probiotics loaded into $\mathrm{pH}$-sensitive polymers cannot be released in an acidic $\mathrm{pH}$ environment due to the deprotonated carboxylic acids in the polymers [14]. However, because these $\mathrm{pH}$ sensitive polymers only have the ability to protect probiotics from harsh gastric conditions, we designed a new type of $\mathrm{pH}$-sensitive polymer using inulin as a prebiotic.

Inulin has been used as a prebiotic source in industrial applications because they are found in many natural sources (e.g., chicory root, Jerusalem artichoke, leek, and onion) [15]. Inulin consists of fructose polymers linked by $\beta(2 \rightarrow 1)$ bonds containing glucosyl moieties at the chain terminal. Due to the $\beta(2 \rightarrow 1)$ linkages in inulin, it is not digested by pancreatic enzymes in the upper GI tract [15], although the gut microbiota can ferment inulin and produce short chain fatty acids (SCFAs), which induces the growth of beneficial microorganisms, thereby altering the composition of organisms in the gut microbiome and boosting the host immune system [16, 17]. Also, there has been a growing interest in the use of inulin as an adjuvant or drug delivery system. Interestingly, delta inulin in microparticle form showed adjuvanting ability for enhancing immune activity in vaccines against influenza, hepatitis B, etc. [18], although soluble inulin has less immunological activity [19].

In the health industry, there are several methods of formulating probiotics for use as food supplements and these include powder, liquid, and spray forms, although the most commonly used is powder. Although there have not been many reports, on creating a tablet form for oral delivery of probiotics one previous study did show that it is easy to formulate probiotics into tablet form with a $\mathrm{pH}$ sensitive polymer that successfully protected probiotics from harsh stomach conditions [20].

In this study however, we aimed to develop a new $\mathrm{pH}-$ sensitive tablet using phthalyl inulin (PI) to protect the L. reuteri LRT18 (LR) from harsh gastric conditions.
Moreover, we obtained promising results for further in vivo application. To the best of our knowledge, this is the first report to exhibit the $\mathrm{pH}$-sensitive properties of PI that protect probiotics from harsh gastric conditions.

\section{Materials and Methods}

\section{Materials \\ L. reuteri LRT18 (LR, KCTC3594) used in this study was isolated from a previous study [5]. All of the materials and chemicals used in this study were purchased from Sigma-Aldrich (USA) unless otherwise stated. De Man, Rogosa and Sharpe agar (MRS) broth and MRS agar were purchased from BD Difco (Sparks, USA) for the bacterial cultures.}

\section{Synthesis of Phthalyl Inulin (PI)}

Phthalyl inulin (PI) was synthesized as described in a previous report [21]. Briefly, $1 \mathrm{~g}$ of inulin (MW: 5,000 g/mol) was dissolved in $5 \mathrm{ml}$ of $\mathrm{N}, \mathrm{N}$-dimethylformamide and $2.0 \mathrm{~g}$ of phthalic anhydride were added in the above solution and $0.2 \mathrm{ml}$ of $5 \%(\mathrm{w} / \mathrm{v})$ sodium acetate was used as a catalyst. The reaction was conducted at $40^{\circ} \mathrm{C}$ for $24 \mathrm{~h}$ under nitrogen gas. And then, the PI was dialyzed in cold water for $24 \mathrm{~h}$. The PI was lyophilized and stored at $-20^{\circ} \mathrm{C}$ until use. The conjugation of phthalyl groups in PI was confirmed by $600 \mathrm{MHz}{ }^{1} \mathrm{H}-\mathrm{NMR}$ spectroscopy (AVANCE600, Bruker, Germany).

\section{Tablet Preparation}

LR cultures were grown in MRS broth at $37^{\circ} \mathrm{C}$ for $24 \mathrm{~h}$ and collected by centrifugation. Harvested cells were washed 3 times in phosphate buffer solution and suspended in $10 \%$ skim milk. The cells were then frozen at $-20^{\circ} \mathrm{C}$ for $12 \mathrm{~h}$ and lyophilized. The lyophilized probiotics were ground into a fine powder and stored at $4^{\circ} \mathrm{C}$ until use. The tablets were prepared at room temperature (RT) by direct compression using a single press. For the tablets, a mixture of LR and PI (weight ratio of LR to PI = 1:1) was filled into a $4 \mathrm{~mm}$ diameter die. The tablets were formed under different pressures ranging from 3 to 10 kilopascal (KP) with a plane surface according to Tao et al. [20].

\section{Measurement of the Probiotic (LR) Viability and Disintegration Time of Tablets}

The viability of LR in the tablet was expressed as colony forming units (CFU). Briefly, the tablets were broken and dispersed in $1 \mathrm{ml}$ of phosphate buffer solution (PBS, pH 6.8). And then, the serial-diluted suspension was dropped into the MRS agar plate and incubated at $37^{\circ} \mathrm{C}$ to count the LR colonies according to the Tao et al. method [22]. The tablets were transferred into $5 \mathrm{ml}$ PBS ( $\mathrm{pH}$ 6.8) and the complete disintegration time was measured.

\section{Measurement of the Swelling Ratio of Tablets}

The tablets were transferred into $5 \mathrm{ml}$ simulated gastric fluid (SGF) adjusted to $\mathrm{pH} 2$ with pepsin $(1,000 \mathrm{U} / \mathrm{ml})$. The swelling ratio was calculated by the following equation [22]. 


$$
\mathrm{Q}=\left(\mathrm{M}_{\mathrm{s}}-\mathrm{M}_{\mathrm{d}}\right) / \mathrm{M}_{\mathrm{d}}
$$

The swelling ratio is $Q$, the $M_{d}$ is the tablet mass in the dried state and the $\mathrm{M}_{\mathrm{s}}$ is the mass of the tablet in the swollen state. At the beginning of the experiment, the excess water outside the tablet was removed.

\section{Stability of the Tablets in the SGF with or without Pepsin}

The stability studies were performed as described in a previous method [20]. The SGF was prepared by PBS adjusted to $\mathrm{pH} 2.0$ with or without pepsin $(1,000 \mathrm{U} / \mathrm{ml})$ by $1 \mathrm{M} \mathrm{HCl}$. The tablets and LR powder were transferred into $5 \mathrm{ml}$ of SGF with or without pepsin. The survivability of LR was observed as the CFU at the end of the incubation period $(0,30,60,90,120 \mathrm{~min})$ when incubated at $37^{\circ} \mathrm{C}$ with $100 \mathrm{rpm}$.

Viability of the Tablets in SGF and Simulated Intestinal Fluid (SIF) Medium in Sequential Exposure

The cell viability of LR in the tablets sequentially exposed to SGF and SIF was performed by the following method [20] with some modifications. Tablets were incubated in $5 \mathrm{ml} \mathrm{SGF} \mathrm{(pH} \mathrm{2,}$ $1,000 \mathrm{U} / \mathrm{ml}$ pepsin) at $37^{\circ} \mathrm{C}$ with $100 \mathrm{rpm}$ for $2 \mathrm{~h}$. Then, the tablets were quickly transferred to $5 \mathrm{ml} \mathrm{SIF}$ and incubated at $37^{\circ} \mathrm{C}$ with $100 \mathrm{rpm}$ for $4 \mathrm{~h}$. SIF was prepared by PBS adjusted to $\mathrm{pH} 6.8$ with $1.2 \%(\mathrm{w} / \mathrm{v})$ bile salt. The viable cells were counted in the supernatant medium as were the non-disintegrated tablets at each incubation time.

\section{Tablet Stability}

The stability of the tablets was tested when stored at $4^{\circ} \mathrm{C}$ for up to 6 months. Every month the cell viability was counted as described above.

\section{Statistical Analysis}

Data are presented as the mean \pm SEM of three independent experiments. The statistical significance was analyzed between each group by one-way ANOVA and Tukey's test $\left({ }^{*} p<0.05\right.$; $\left.{ }^{* *} p<0.01,{ }^{* * *} p<0.001\right)$.

\section{Results}

\section{Synthesis and Characterization of PI}

To develop a $\mathrm{pHs}$ sensitive polymer, the phthalic group was introduced to inulin by an ester bond between hydroxyl groups in inulin and carboxylic acids in phthalic acid. The reaction scheme of the synthesis of PI is shown in Fig. 1A. After synthesizing the PI, the degree of the phthalic groups in the PI was estimated by measurement of ${ }^{1} \mathrm{H}-\mathrm{NMR}$. The fifth protons of inulin appeared at $3.8 \mathrm{ppm}$

A

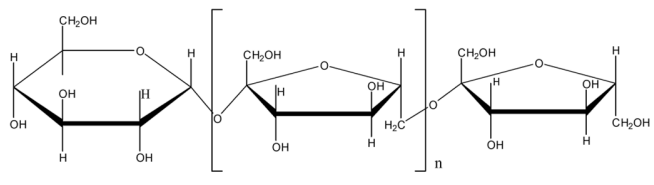

Inulin from chicory (INL)<smiles>O=C1OC(=O)c2ccccc21</smiles>

DMF

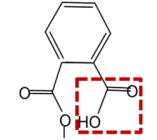
pH sensitivity
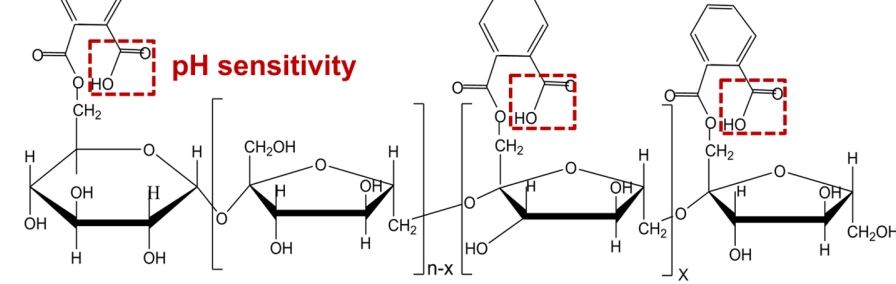

Phthalyl inulin

(PI)

Phthalic anhydride

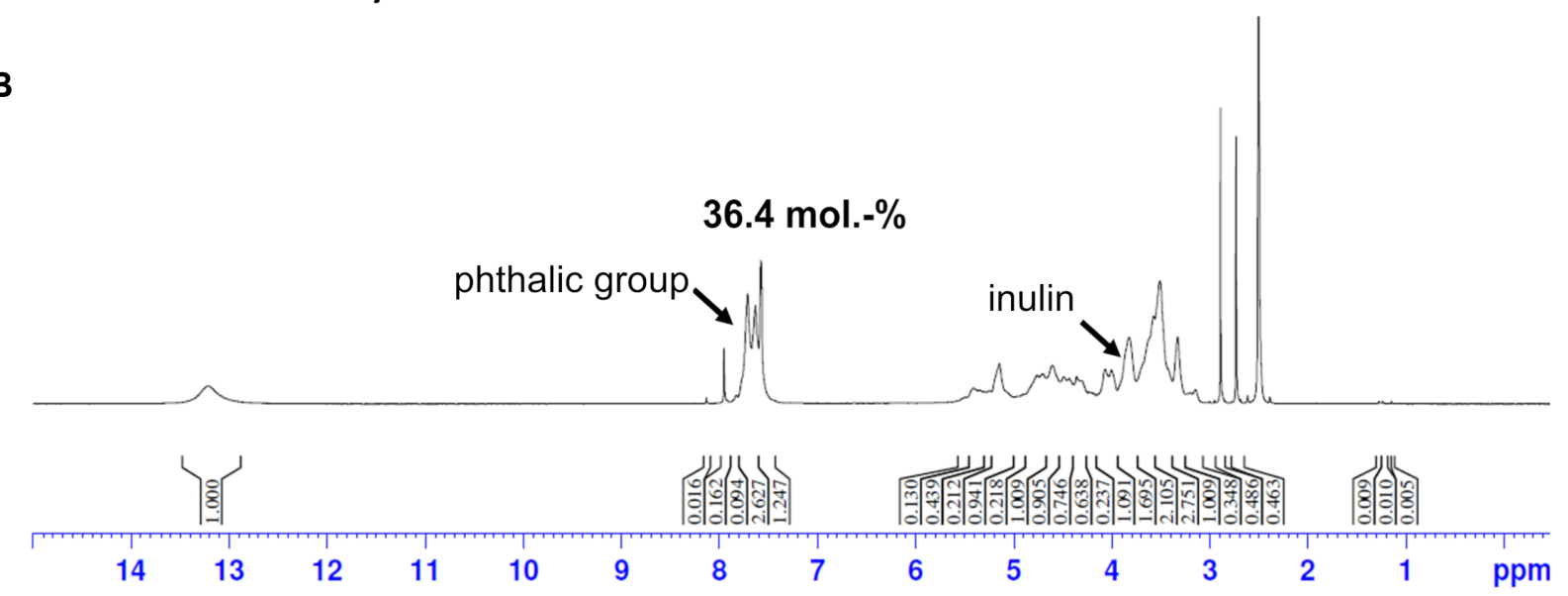

Fig. 1. Scheme and characterization of phthalyl inulin (PI). Chemical reaction scheme of PI (A) and NMR spectrum of PI (B). 


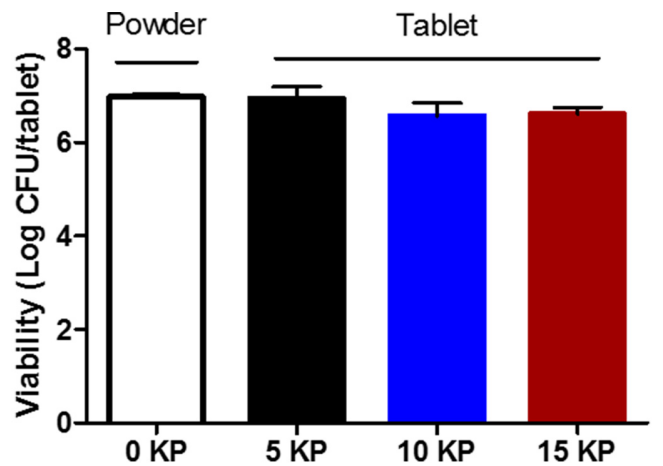

Fig. 2. Viability of L. reuteri (LR) after tableting under different compression forces $(5,10$, and $15 \mathrm{KP}$ ) (means \pm standard deviation, $\mathrm{SD} ; n=3)$.

and the protons of the phthalic groups in the PI appeared at 7.4-7.7 ppm as shown in Fig. 1B. Based on the integration of protons in both the inulin and phthalic groups, the degree of the phthalic groups in the PI was 36.4 mol.- $\%$.

\section{Effects of Compression Forces on the Viability of LR and Tablet Properties}

To evaluate whether different compression force can affect the viability of LR, we measured it after tableting and found that there were no significant differences in the viability of LR in the tablets even with the use of varied compression forces (Fig. 2). To determine the protective effect of the LR in gastric conditions, the swelling ratio of the tablets and viability of the LR in SGF were measured. The swelling ratio of the tablets prepared according to different compression forces was very low in SGF conditions (Fig. 3). It was observed that the tablets were not completely

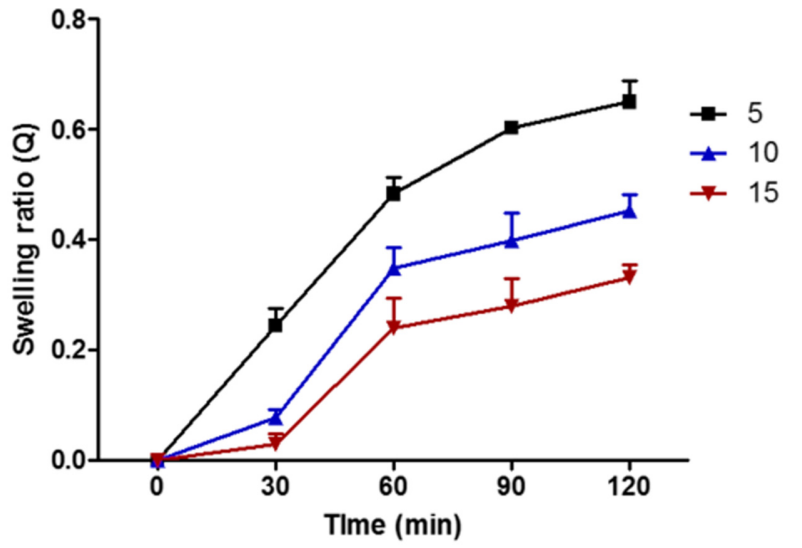

Fig. 3. Swelling ratio of LR-loaded PI tablets with different compression conditions $(5,10$, and $15 \mathrm{KP})$ until 2-h incubation in SGF (means \pm standard deviation, SD; $n=3$ ).

(PI: phthalyl inulin, LR: Lactobacillus reuteri, and SGF: simulated gastric fluid)

disintegrated within $2 \mathrm{~h}$ in gastric conditions. In particular, among the groups, the highest compression force (15 KP) showed the lowest swelling ratio. The viability of LR in gastric conditions was then measured using the SGF conditions with or without pepsin (Fig. 4). The 5, 10, and $15 \mathrm{KP}$ tablets and probiotics alone (powder) were loaded in SGF for $2 \mathrm{~h}$. The results showed that the viability of the probiotics alone dramatically decreased in both the SGF conditions and especially in the presence of pepsin. However, LR-loaded PI tablets were able to protect probiotic death in the SGF regardless of the presence of pepsin. The viability of LR between loaded tablets and LR alone showed significant differences in SGF in the presence of pepsin after $2 \mathrm{~h}$ (Fig. 4B), suggesting that the PI tablets
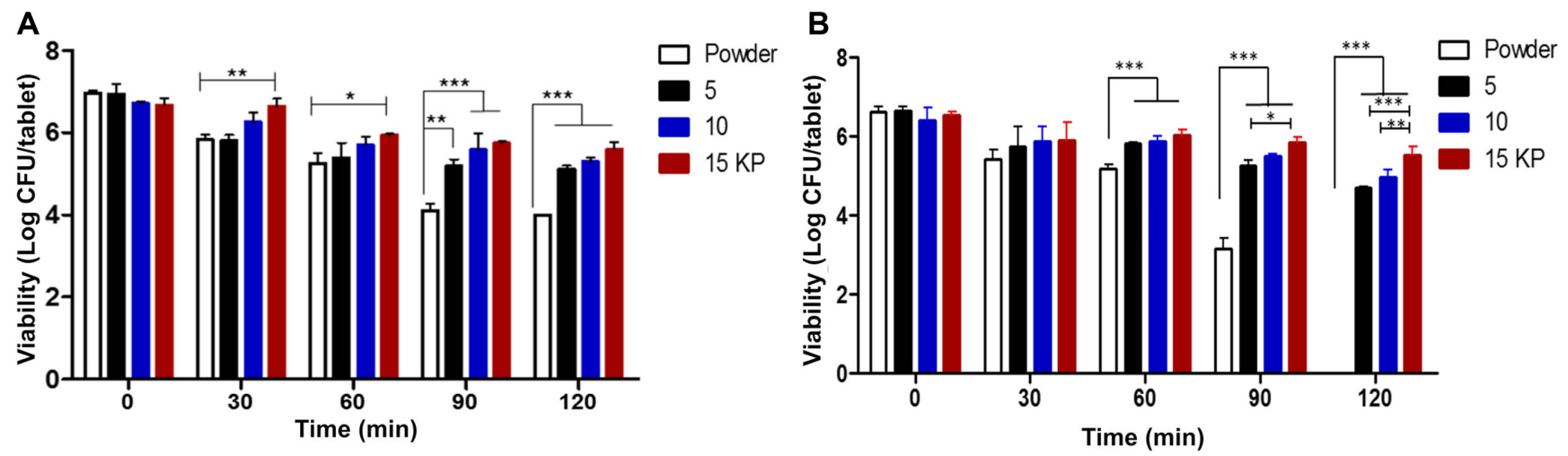

Fig. 4. Survivability of LR in LR-loaded PI tablets in SGF (pH 2.0) without pepsin (A) and with pepsin (B) until $2 \mathrm{~h}$ at $37^{\circ} \mathrm{C}$ (means \pm standard deviation, $\mathrm{SD} ; n=3$ ).

(PI: phthalyl inulin, LR: Lactobacillus reuteri, and SGF: simulated gastric fluid) 


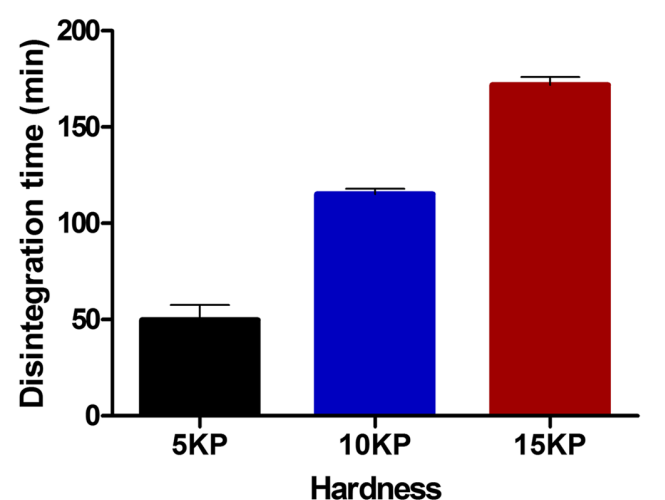

Fig. 5. Disintegration time of LR-loaded PI tablets with different compression forces in PBS ( $\mathrm{pH}$ 6.8) (means \pm standard deviation, $\mathrm{SD} ; n=3)$.

(PI: phthalyl inulin, LR: Lactobacillus reuteri, and PBS: phosphate buffer solution)

were able to protect LR from harsh stomach conditions. Furthermore, the higher compression force increased the viability of the LR in gastric conditions. The LR viability in the $15 \mathrm{KP}$ tablets was significantly higher than LR alone in SGF without pepsin at $30 \mathrm{~min}$ (Fig. 4A). In SGF with pepsin, the difference in viability of LR between LR alone and the LR loaded into tablets, in case of the $15 \mathrm{KP}$ tablet, was significantly higher at $60 \mathrm{~min}$ (Fig. 4B). Moreover, the viability of LR in the $15 \mathrm{KP}$ tablet was significantly higher than LR in the $5 \mathrm{KP}$ and $10 \mathrm{KP}$ tablets after $2 \mathrm{~h}$, especially in the presence of pepsin, indicating that higher compression force was able to protect the probiotics better in gastric conditions.

To investigate the swelling effect in intestinal fluid, times for complete disintegration in SIF were measured among the different compression forces. In Fig. 5, the disintegration time increased with an increase of the compression force. The disintegration time for $15 \mathrm{KP}$ was $160 \mathrm{~min}$; however, for $10 \mathrm{KP}$ it was nearly $110 \mathrm{~min}$ and for $5 \mathrm{KP}$ it was $50 \mathrm{~min}$ or less. This demonstrates that tablets fully disintegrate in intestinal conditions due to the $\mathrm{pH}$ sensitivity of the PI, and also that the compression force affected the disintegration ability of the $\mathrm{pH}$-sensitive tablets.

\section{Release and Viability of LR from LR-Loaded PI Tablets in SGF and SIF}

The release and cell viability of LR from LR-loaded PI tablets in SGF and SIF were tested by sequentially immersing the tablets into both types of fluid. First, as shown in Fig. 6A, the release of LR from the tablets in SGF and SIF was analyzed. In SGF, the viability of the probiotics alone dramatically decreased and no viable cells were found after $2 \mathrm{~h}$ incubation. From the tablets, no viable released cells were found in SGF. In SIF, the $5 \mathrm{KP}$ and 10 $\mathrm{KP}$ tablets released viable cells faster than the $15 \mathrm{KP}$ tablets. The higher compression force tablet delayed the release of LR by comparison with the other two tablets. However, nearly all of the probiotics were released from all tablets of each type after $5 \mathrm{~h}$ of immersion in SIF. Next, the viability of LR was measured by sequentially exposing the tablets to SGF and SIF (Fig. 6B). The viability of the probiotics alone was non-existent after exposure to SGF for $2 \mathrm{~h}$. When the tablets were exposed to SGF, the LR became less viable with time. Although the LR viability slightly changed in SIF after $5 \mathrm{~h}$, more viable cells remained inside the tablets prepared with the higher compression force than with lower compression force. The viability of LR within the $15 \mathrm{KP}$ tablets was significantly higher than the other two groups after $7 \mathrm{~h}$ in SGF and SIF conditions. Altogether, owing to the $\mathrm{pH}$-sensitivity of the PI that was used, the PI tablets
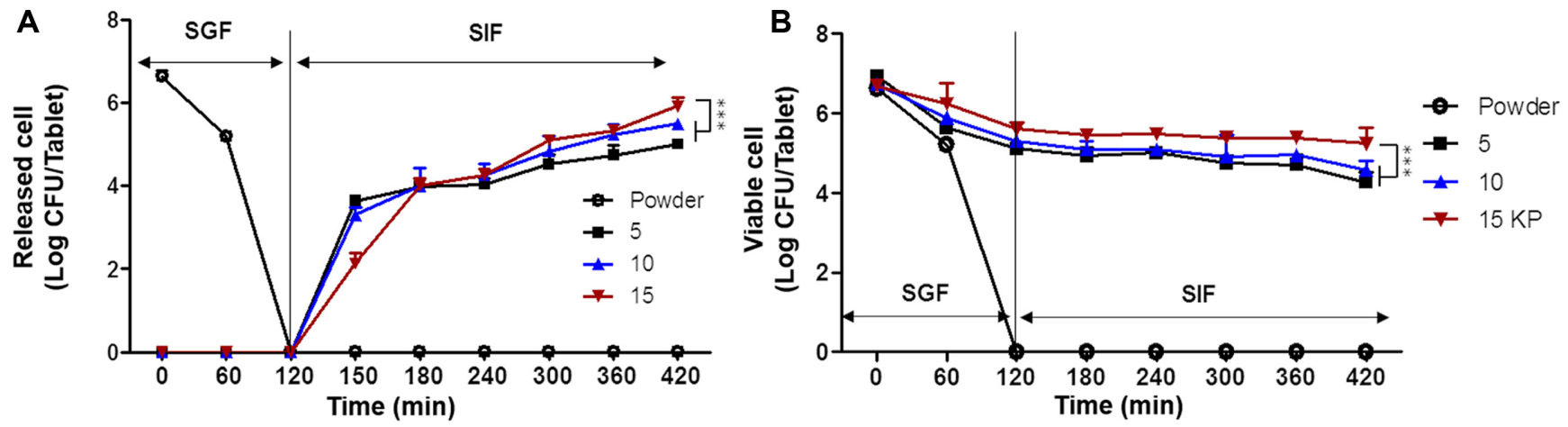

Fig. 6. Released (A) and viable cell numbers (B) of LR from powder and LR-loaded PI tablets sequentially exposed to SGF and SIF until $7 \mathrm{~h}$ at $37^{\circ} \mathrm{C}$ (means \pm standard deviation, SD; $n=3$ ).

(PI: phthalyl inulin, LR: Lactobacillus reuteri, SGF: simulated gastric fluid, and SIF: simulated intestinal fluid) 


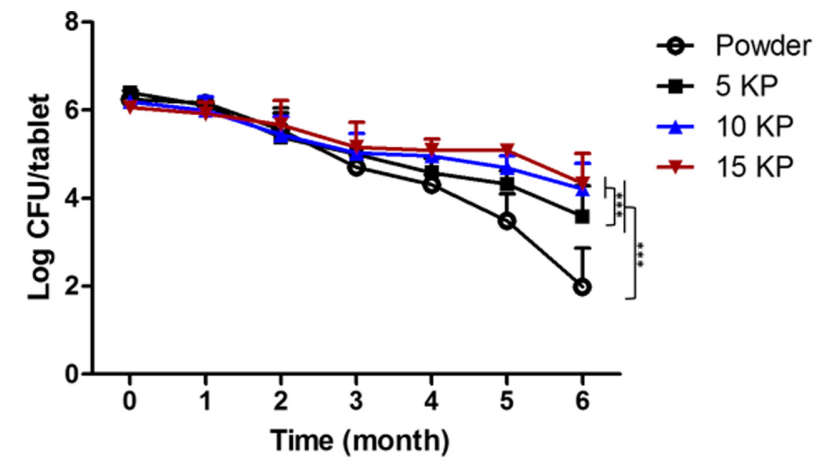

Fig. 7. Storage stability of LR in LR-loaded PI tablets during 6 months at $4^{\circ} \mathrm{C}$ (means \pm standard deviation, SD; $n=3$ ). (PI: phthalyl inulin and LR: Lactobacillus reuteri)

were able to protect LR from harsh gastric conditions and release LR without the LR viability being affected by the PI tablets' disintegration.

\section{LR Viability in LR-Loaded PI Tablets in Long-Term Storage}

The stable viability of probiotics is a major index for the industrialization of probiotics. The stability of LR inside the tablets during storage was checked over a 6-month period at $4^{\circ} \mathrm{C}$. The temperature was selected because most probiotic products recommend storage in a refrigerator. In Fig. 7, the stability of LR for 6 months was calculated by the viable CFU per one tablet. Probiotics in powder form dramatically decreased in viability after 3 months. However, the viability of the LR in tablet form was significantly more stable even after 6 months. In particular, higher compression force tablets (10 and $15 \mathrm{KP})$ showed a significantly higher number of viable cells at the end of 6 months than the $5 \mathrm{KP}$ tablet. Overall, the viability of LR was more stable in PIbased tablets while higher compression force also allowed for higher cell stability.

\section{Discussion}

In this study, we developed a new $\mathrm{pH}$-sensitive tablet using PI and examined its potential usage and effect on probiotic survivability and stability for oral delivery of probiotics. In delivering probiotics to the host gut, one of the most important aspects is to permit the probiotics to survive after passing through harsh gastric conditions [23]. Generally, most probiotic products on the market for human use are now sold in liquid or semi-liquid forms containing probiotics and prebiotics together [24]. Such products provide only low cell viability after oral ingestion because prebiotics cannot protect probiotics from harsh gastric conditions in mixture form [24]. Furthermore, the probiotics used for livestock animals are administered orally through feed although most probiotics are just added to feed without any protection and the amount of the probiotics used is very inconsistent from animal to animal [25]. Therefore, developing a dry form of probiotics that can protect the microorganisms from harsh stomach conditions and homogenous administration of the probiotics through the oral route are needed. For these reasons, we designed a new $\mathrm{pH}$-sensitive PI to protect probiotics in low $\mathrm{pH}$ conditions and to form homogenous tablets as a dry form for oral administration. The PI was prepared by conjugation with phthalic anhydride with inulin through ester bond linkage because the remained carboxylic acid groups in phthalic acids after conjugation reaction have $\mathrm{pH}$-sensitive properties due to the deprotonation at $\mathrm{pH} 7$ and protonation in low $\mathrm{pH}$ (such as $\mathrm{pH}$ 2) [26], which is similar with CAP or HPMCP used for popular oral delivery systems [11, 27, 28].

Inulin has been widely used as a prebiotic for many decades because it promotes the growth and activity of probiotic L. reuteri [29]. It has also been used in drug delivery systems for intranasal, parenteral, intravenous, and subcutaneous routes of administration [30] although inulin itself is difficult to use as a carrier in an oral colonic drug delivery system because inulin is highly soluble in water. Therefore, many strategies have been tried to reduce the solubility of inulin in water by mixing hydrophobic coating materials such as Eudragit [31, 32] or by conjugating hydrophobic residues [33]. By conjugation of the phthalyl groups to inulin, we were able to reduce its water solubility. The $\mathrm{pH}$-sensitive PI protected the probiotics from the low $\mathrm{pH}$ condition and released them after the dissolution of polymers in neutral $\mathrm{pH}$ condition. Moreover, in our previous study it was found that phthalyl inulin nanoparticles as a new type of prebiotics were able to enhance the antimicrobial activity of probiotics [21]. Therefore, we can assume that PI would have multi-functional properties when it is orally administered. Firstly, PI can be used as a tablet material to protect probiotics from harsh gastric conditions as previously mentioned. Secondly, PI may enhance the growth and activity of probiotics after the hydrolysis of PI into inulin and degradation of inulin into fructooligosaccharides (FOS) in the host gut. Thirdly, PI may also enhance the antimicrobial activity of probiotics when phthalyl inulin nanoparticles are mixed with the PI tablet. However, more study on this concept should be conducted in the near future.

In our previous result, we isolated L. reuteri LRT18 (LR) from porcine feces and it showed the highest antimicrobial 
activities against both pathogenic bacteria K88-positive E. coli and S. enterica subsp [5], although it was very weak against acid stress, especially in the presence of pepsin (Fig. 4). Therefore, we used $\mathrm{pH}$-sensitive PI to protect LR from harsh acidic conditions and deliver LR to the host gut efficiently. First, we investigated the LR survival during tableting under different compression forces. There were no significant differences in probiotic viability among the different compression forces, which was similar to previous results $[20,34]$. The LR viability in the $15 \mathrm{KP}$ tablet received the most protective effect even after $2 \mathrm{~h}$ of incubation in the SGF condition. For the incubation in SGF with or without pepsin, the survivability of LR in the $15 \mathrm{KP}$ tablet was both approximately 7 Log CFU. Especially, the LR viability loaded in the $15 \mathrm{KP}$ tablet was significantly higher than with other tablet groups in SGF with pepsin. The results suggest that when tableting probiotics, compression force is one of the important factors in protecting probiotics because a high compression force prohibits the fluid from physically coming inside the tablets. Moreover, the results were also consistent with the different disintegration times and swelling ratio between the groups. The disintegration and swelling ratio of the tablets in $\mathrm{pH} 2$ are shown in Fig. 3. It was found that the swelling ratios of tablets were different according to the compression forces although the ratios in all three groups were very low in SGF conditions for $2 \mathrm{~h}$ incubation, suggesting that this was due to the $\mathrm{pH}$ sensitivity of the tablets. Furthermore, in Fig. 5, the disintegration times of tablets in SIF conditions differed from their compression forces. Higher compression forces meant more time for the tablets to disintegrate. The results were consistent with the release behavior in SIF conditions in Fig. 6, where the $15 \mathrm{KP}$ tablet released LR more slowly than 5 or $10 \mathrm{KP}$ tablets for more than $60 \mathrm{~min}$ after changing to SIF conditions. However, total viable cells released from the tablets were significantly higher in $15 \mathrm{KP}$ than 5 or $10 \mathrm{KP}$ because the survival rate of $\mathrm{LR}$ in $15 \mathrm{KP}$ tablets was higher after incubation in SGF conditions.

To check the $\mathrm{pH}$-sensitivity of the PI, we incubated the PI tablets in the SGF media for $2 \mathrm{~h}$ with or without pepsin and compared them with LR alone (powder). Also, we exposed the PI tablets sequentially to SGF and SIF media. For identifying the survivability of probiotics in the gastrointestinal tract, most researchers choose $\mathrm{pH}$ 1.5-2 for gastric conditions and $\mathrm{pH}$ 6.8-7.2 for intestinal conditions because the presence of pepsin and low $\mathrm{pH}$ are required to represent the stomach environment when testing the survivability of probiotics $[35,36]$. Although the $\mathrm{pH}$ of the stomach slowly declines from a neutral $\mathrm{pH}$ to $\mathrm{pH} 2$ when food is ingested [37, 38], the $\mathrm{pH}$ of the stomach during fasting can decrease to 1.5, which suggests that an acidic environment is highly challenging for probiotic survival when administered orally [39]. Also, it has been generally reported that ingested food remains in the stomach for 2$3 \mathrm{~h}$ and transits to the intestinal tract where it then remains for 5-12 h [40], although bile salt may have an antimicrobial effect toward bacteria, intestinal $\mathrm{pH}$ is known to be $\mathrm{pH}$ 6.8-7.2, which is more suitable for bacteria to survive [39]. Therefore, tablets should be effective in protecting probiotics in gastric conditions throughout this time while releasing the probiotics in the intestine. The survivability of LR in the PI tablets was significantly higher than LR alone for $30 \mathrm{~min}$ incubation in SGF media with or without pepsin, indicating PI tablets were able to protect LR from acidic conditions. The swelling degree of PI tablet in SIF was higher than in SGF due to the $\mathrm{pH}$ sensitivity of the PI. Moreover, the viability and release behavior of LR in SGF and SIF were shown to be similar to other types of $\mathrm{pH}$-sensitive tablets [41]. In SGF the viable released cells were not shown and the fast release of probiotics was shown in SIF as the swelling degree of PI increases at $\mathrm{pH}$ 6.8. Even though higher compression force delayed the release of LR more than $1 \mathrm{~h}$ in SIF, after $5 \mathrm{~h}$ incubation all tablets viable released cell counts had the same viable cell counts as Fig. 6B in SIF. This can indicate that LR is able to be delivered and work in the intestine since in ingested food generally remains there for up to $12 \mathrm{~h}$. However, our study may have limitations in in vitro conditions because it is very difficult to mimic in vivo conditions by adjusting the $\mathrm{pH}$ with enzymes. The $\mathrm{pH}$ in the digestive system actually declines slowly from neutral to $\mathrm{pH} 2$ over the course of $3 \mathrm{~h}$ after a meal. Therefore, a follow-up study on the protection and release of LR from the new type of $\mathrm{pH}$-sensitive tablets should be conducted in vivo in the near future.

LR-loaded PI tablets also increased LR stability for longterm storage compared to LR alone. The results suggest that PI tablets can be used in industry since many probiotics are stored at $4^{\circ} \mathrm{C}$ for more than three months. In conclusion, PI is a suitable material for making probiotic tablets that can preserve cells in harsh gastric conditions, release easily in the intestinal condition and show longterm stable storage. To the best of our knowledge, this is the first report to suggest the possibility of PI as a tableting material and to be used as an alternative to antibiotics in industry. 


\section{Acknowledgments}

This study was supported by a grant from the Strategic Initiative for Microbiomes in Agriculture and Food, Ministry of Agriculture, Food and Rural Affairs (MAFRA), Republic of Korea, as part of the Post Genome Joint Department Genome Project (Grant ID: 914005-04) and was supported by the Basic Science Research Program through the National Research Foundation of Korea (NRF) funded by the Ministry of Education (No. 2016936920) and BK21 Plus Program of the Department of Agricultural Biotechnology, Seoul National University, Seoul, Korea.

\section{Conflict of Interest}

The authors have no financial conflicts of interest to declare.

\section{References}

1. Kechagia M, Basoulis D, Konstantopoulou S, Dimitriadi D, Gyftopoulou K, Skarmoutsou N, et al. 2013. Health benefits of probiotics: a review. ISRN Nutr. 2013: 481651.

2. Forkus B, Ritter S, Vlysidis M, Geldart K, Kaznessis YN. 2017. Antimicrobial probiotics reduce salmonella enterica in turkey gastrointestinal tracts. Sci. Rep. 7: 40695.

3. Doyle MP, Erickson MC. 2006. Reducing the carriage of foodborne pathogens in livestock and poultry. Poult. Sci. 85: 960-973.

4. Dowarah R, Verma AK, Agarwal N. 2017. The use of Lactobacillus as an alternative of antibiotic growth promoters in pigs: a review. Anim. Nutr. 3: 1-6.

5. Lee JY, Han GG, Choi J, Jin GD, Kang SK, Chae BJ, et al. 2017. Pan-genomic approaches in lactobacillus reuteri as a porcine probiotic: investigation of host adaptation and antipathogenic activity. Microb. Ecol. 74: 709-721.

6. Sinha VR, Kumria R. 2001. Polysaccharides in colon-specific drug delivery. Int. J. Pharm. 224: 19-38.

7. Akhgari A. 2015. Role of polysaccharides in colon-specific drug delivery. Jundishapur. J. Nat. Pharm. Prod. 10: e30388.

8. Ravi V, Kumar STMP. 2008. Influence of natural polymer coating on novel colon targeting drug delivery system. J. Mater. Sci. Mater. Med. 19: 2131-2136.

9. Papadimitriou K, Zoumpopoulou G, Foligne B, Alexandraki V, Kazou M, Pot B, et al. 2015. Discovering probiotic microorganisms: in vitro, in vivo, genetic and omics approaches. Front. Microbiol. 6: 58.

10. Petros RA, DeSimone JM. 2010. Strategies in the design of nanoparticles for therapeutic applications. Nat. Rev. Drug Discov. 9: 615-627.

11. Singh B, Maharjan S, Jiang T, Kang SK, Choi YJ, Cho CS. 2015. Attuning hydroxypropyl methylcellulose phthalate to oral delivery vehicle for effective and selective delivery of protein vaccine in ileum. Biomaterials 59: 144-159.

12. Fukui E, Miyamura N, Kobayashi M. 2001. An in vitro investigation of the suitability of press-coated tablets with hydroxypropylmethylcellulose acetate succinate (HPMCAS) and hydrophobic additives in the outer shell for colon targeting. J. Control. Release 70: 97-107.

13. Lee HB, Yoon SY, Singh B, Oh SH, Cui LH, Yan CG, et al. 2018. Oral immunization of FMDV vaccine using $\mathrm{pH}$ sensitive and mucoadhesive thiolated cellulose acetate phthalate microparticles. Tissue Eng. Regen. Med. 15: 1-11.

14. Liu L, Yao WD, Rao YF, Lu XY, Gao JQ. 2017. pHResponsive carriers for oral drug delivery: challenges and opportunities of current platforms. Drug Deliv. 24: 569-581.

15. Mensink MA, Frijlink HW, Maarschalk KV, Hinrichs WLJ. 2015. Inulin, a flexible oligosaccharide I: review of its physicochemical characteristics. Carbohydr. Polym. 130: 405419.

16. Tremaroli V, Backhed F. 2012. Functional interactions between the gut microbiota and host metabolism. Nature 489: 242-249.

17. Seifert S, Watzl B. 2007. Inulin and oligofructose: review of experimental data on immune modulation. J. Nutr. 137: 2563s-2567s.

18. Skwarczynski M. 2017. Inulin: a new adjuvant with unknown mode of action. EBIO Medicine 15: 8-9.

19. Petrovsky N, Cooper PD. 2015. Advax, a novel microcrystalline polysaccharide particle engineered from delta inulin, provides robust adjuvant potency together with tolerability and safety. Vaccine 33: 5920-5926.

20. Jiang T, Li HS, Han GG, Singh B, Kang SK, Bok JD, et al. 2017. Oral delivery of probiotics in poultry using $\mathrm{pH}$ sensitive tablets. J. Microbiol. Biotechnol. 27: 739-746.

21. Kim W-S, Lee J-Y, Singh B, Maharjan S, Hong L, Lee S-M, et al. 2018. A new way of producing pediocin in Pediococcus acidilactici through intracellular stimulation by internalized inulin nanoparticles. Sci. Rep. 8: 5878.

22. Chavda H, Patel C. 2011. Effect of crosslinker concentration on characteristics of superporous hydrogel. Int. J. Pharm. Investig. 1: 17-21.

23. Solanki HK, Pawar DD, Shah DA, Prajapati VD, Jani GK, Mulla AM, et al. 2013. Development of microencapsulation delivery system for long-term preservation of probiotics as biotherapeutics agent. Biomed. Res. Int. 2013: 620719.

24. Collins JW LRR, Woodward MJ, Searle LE 2009. pp. 11231192. Application of Prebiotics and Probiotics in Livestock. Prebiotics and probiotics science and technology. Springer, BerlinHeideberg. Germany.

25. Cheng G, Hao H, Xie S, Wang X, Dai M, Huang L, et al. 2014. Antibiotic alternatives: the substitution of antibiotics in animal husbandry? Front. Microbiol. 5: 217.

26. Yang $X Y$, Wang $Y S$, Huang $X$, Ma YF, Huang $Y$, Yang RC, et al. 2011. Multi-functionalized graphene oxide based 
anticancer drug-carrier with dual-targeting function and pH-sensitivity. J. Mater. Chem. 21: 3448-3454.

27. Wang $X Q$, Zhang Q. 2012. pH-sensitive polymeric nanoparticles to improve oral bioavailability of peptide/ protein drugs and poorly water-soluble drugs. Eur. J. Pharm. Biopharm. 82: 219-229.

28. Dai JD, Nagai $T$, Wang $X Q$, Zhang $T$, Meng $M$, Zhang $Q$. 2004. $\mathrm{pH}$-sensitive nanoparticles for improving the oral bioavailability of cyclosporine A. Int. J. Pharm. 280: 229-240.

29. Oliveira RPD, Perego P, de Oliveira MN, Converti A. 2012. Effect of inulin on the growth and metabolism of a probiotic strain of Lactobacillus rhamnosus in co-culture with Streptococcus thermophilus. Lwt-Food Sci. Technol. 47: 358-363.

30. Imran S, Gillis RB, Kok MS, Harding SE, Adams GG. 2012. Application and use of Inulin as a tool for therapeutic drug delivery. Biotechnol. Genet. Eng.Rev. 28: 33-45.

31. Akhgari A, Farahmand F, Afrasiabi Garekani H, Sadeghi F, Vandamme TF. 2006. Permeability and swelling studies on free films containing inulin in combination with different polymethacrylates aimed for colonic drug delivery. Eur. J. Pharm. Sci. 28: 307-314.

32. Van den Mooter G, Vervoort L, Kinget R. 2003. Characterization of methacrylated inulin hydrogels designed for colon targeting: In vitro release of BSA. Pharm. Res. 20: 303-307.

33. Castelli F, Sarpietro MG, Micieli D, Ottimo S, Pitarresi G, Tripodo G, et al. 2008. Differential scanning calorimetry study on drug release from an inulin-based hydrogel and its interaction with a biomembrane model: $\mathrm{pH}$ and loading effect. Eur. J. Pharm. Sci. 35: 76-85.
34. Calinescu C, Mulhbacher J, Nadeau E, Fairbrother JM, Mateescu MA. 2005. Carboxymethyl high amylose starch (CM-HAS) as excipient for Escherichia coli oral formulations. Eur. J. Pharm. Biopharm. 60: 53-60.

35. Sahu A, Bora U, Kasoju N, Goswami P. 2008. Synthesis of novel biodegradable and self-assembling methoxy poly(ethylene glycol)-palmitate nanocarrier for curcumin delivery to cancer cells. Acta Biomater. 4: 1752-1761.

36. Lian WC, Hsiao HC, Chou CC. 2003. Viability of microencapsulated bifidobacteria in simulated gastric juice and bile solution. Int. J. Food Microbiol. 86: 293-301.

37. Debas HT. 1977. Regulation of gastric acid secretion. Fed. Proc. 36: 1933-1937.

38. Holzapfel WH, Haberer P, Snel J, Schillinger U, Huis in't Veld JH. 1998. Overview of gut flora and probiotics. Int. J. Food Microbiol. 41: 85-101.

39. Masco L, Crockaert C, Van Hoorde K, Swings J, Huys G. 2007. In vitro assessment of the gastrointestinal transit tolerance of taxonomic reference strains from human origin and probiotic product isolates of Bifidobacterium. J. Dairy Sci. 90: 3572-3578.

40. Sorensen TL, Blom M, Monnet DL, Frimodt-Moller N, Poulsen RL, Espersen F. 2001. Transient intestinal carriage after ingestion of antibiotic-resistant Enterococcus faecium from chicken and pork. N. Engl. J. Med. 345: 1161-1166.

41. Anal AK, Singh H. 2007. Recent advances in microencapsulation of probiotics for industrial applications and targeted delivery. Trends Food Sci. Tech. 18: 240-251. 